\title{
Robotic modified radical hysterectomy with pelvic lymphadenectomy
}

\author{
A Maggioni, L Bocciolone, M Peiretti, F Landoni, V Zanagnolo, L Minig, G Roviglione and N Colombo
}

Division of Gynecologic Oncology, European Institute of Oncology, Milan, Italy

\begin{abstract}
Radical hysterectomy, the complete removal of a woman's uterus, is usually performed via an abdominal incision that requires a 3-5 day hospital stay and a 6-8 week recovery period. Now, in a handful of hospitals around the world, new robotic technology allows doctors to perform this procedure through small incisions that require a recovery time of only one night in the hospital and a significantly shorter recovery period at home. Watch such a procedure being carried out at the European Institute of Oncology.
\end{abstract}

Published: 20/09/2007

Received: 29/07/2007

ecancer 2007, 1:55 DOI: 10.3332/ecancer.2008.55

Copyright: (c) the authors; licensee ecancermedicalscience. This is an Open Access article distributed under the terms of the Creative Commons Attribution License (http://creativecommons.org/licenses/by/2.0), which permits unrestricted use, distribution, and reproduction in any medium, provided the original work is properly cited.

Competing Interests: The authors have declared that no competing interests exist. 


\section{Objective}

To report the feasibility and safety of robotic modified radical hysterectomy and bilateral pelvic lymphadenectomy, using the four-arm da Vinci robot system, in a patient with early-stage cervical carcinoma.

\section{Methods}

The patient is a 37-year-old woman P0G0, who was diagnosed with a severe cervical dysplasia on a pap smear during a regular follow-up check-up visit. She underwent a biopsy that revealed a grade three invasive cervical squamous carcinoma, and she was referred to our institute. The computerized tomography of the abdomen and pelvis was negative for metastatic disease, and MRI of the pelvis revealed a cervical lesion of $16 \times 12 \mathrm{~mm}$. She was therefore offered a radical hysterectomy, using the da Vinci robotic set. She signed an informed consent form, and the risks and benefits of open surgery, versus a minimally invasive procedure, were discussed.

\section{To view this video click here:}

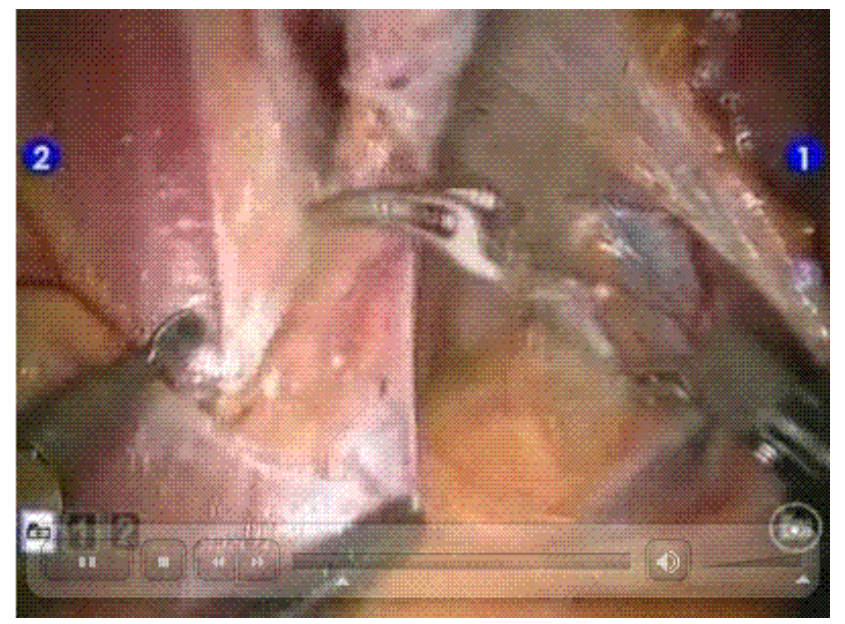

http://www.ecancermedicalscience.com/view-article.asp?doi=10.3332/ecancer.2008.55

\section{Results}

The overall operative time was two hours and 20 minutes, with only two minutes for docking of the robotic arms. Estimated blood loss was $<50 \mathrm{cc}$. There were no intra-operative or postoperative complications. Patient removed Foley catheter on post-operative day three, and she went home the same day with intermittent self-catheterization. Pathological findings revealed a squamous cell carcinoma $\mathrm{G} 3$ with a depth of stromal invasion of $6 \mathrm{~mm}$ and horizontal extension of $12 \mathrm{~mm}$. No lymph vascular spaces involvements were noted. Bilateral parametria and vaginal margins were free of the disease. Twenty-six negative pelvic lymph nodes were removed.

\section{Conclusion}

Modified radical hysterectomy with bilateral pelvic lymphadenectomy using the four-armed da Vinci Robot seems to be a safe and feasible procedure. Surgeons can clearly benefit from better dexterity precision and visualization with the three-dimensional imaging than conventional laparoscopy. Limitations such as the absence of tactile feedback and high costs will need to be addressed from further controlled studies. 